\title{
Properties of young massive clusters obtained with different massive-star evolutionary models.
}

\author{
Aida Wofford and Stéphane Charlot \\ UPMC-CNRS, UMR7095, Institut d'Astrophysique de Paris, F-75014 Paris, France \\ email: wofford@iap.fr, charlot@iap.fr
}

\begin{abstract}
We undertake a comprehensive comparative test of seven widely-used spectral synthesis models using multi-band HST photometry of a sample of eight YMCs in two galaxies. We provide a first quantitative estimate of the accuracies and uncertainties of new models, show the good progress of models in fitting high-quality observations, and highlight the need of further comprehensive comparative tests.
\end{abstract}

Keywords. (ISM:) H II regions - galaxies: star clusters: general - galaxies: star formation ultraviolet: galaxies - stars: early-type.

Overview. Predictions of the integrated light of stellar populations (spectral synthesis models) are crucial input in fields ranging from feedback in cosmological simulations to tools for inferring properties of unresolved stellar populations nearby and at high redshift. Recently, several independent groups have released new models that account for updated input physics (Tang+14 / Charlot \& Bruzual15), the effects of rotation (Georgy+12; Ekström+13 / Leitherer+14), and interacting binaries (Eldridge+08 / Eldridge+15). The references just above are for massive-star evolutionary tracks / population synthesis codes where the tracks are implemented. We compare seven widely-used spectral synthesis models obtained with older and state-of-the-art tracks from the Padova, Geneva, and Auckland groups. We test the models against observations from the Treasury HST program, Legacy ExtraGalactic Ultraviolet Survey (Calzetti+15). Given its high angular resolution, extensive inventory, and inclusion of an NUV band, LEGUS is currently a unique dataset to undertake such study. Our sample is composed of eight available young massive clusters (YMCs) in galaxies NGC $1566(Z=0.014)$ and NGC $5253(Z=0.004)$, which have masses of $\geqslant 5 \times 10^{4} \mathrm{M}_{\odot}$ to mitigate the stochastic sampling of the stellar IMF, and ages of $\leqslant 50 \mathrm{Myr}$ to ensure the presence of massive stars. We use standard models to select the clusters. NGC 1566 was observed in WFC3/UVIS broad band filters F275W (2704 ̊), F336W (3355 ^), F438W (4325 ̊), F555W (5308 $\AA$ ), and F814W $(8024 \AA)$. For NGC 5253, the latter three filters are replaced with archival ACS/HRC observations in close filters F435W (4311 $)$, F550M (5578 $\AA$ ), and F814W $(8115 \AA)$. For the LEGUS cluster catalogue and photometry see Adamo+15. Our models account for stellar and nebular contributions. For the nebulae, we use Cloudy (Ferland+13) and parameters as in Zackrisson+11. For simplicity, we fix the metallicity to mean values of the host galaxies. We try two dust attenuation corrections: starburst (Calzetti+00) and alternative (SMC, Gordon+03, NGC 5253; Milky Way, Mathis+90, NGC 1566). We address 3 questions. 1) How well do models based on state-of-the-art massive-star evolution prescriptions fit the data. 2) Are the properties of YMCs well constrained by the data and models that fit the data. 3) What is the scatter in properties obtained with different models. Our work is discussed in detail in Wofford +15 . 

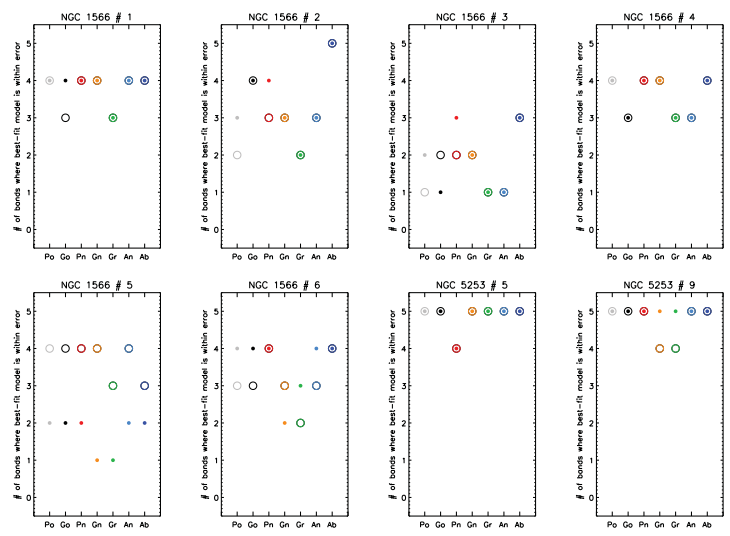

Figure 1. Performance of the different models.

Results. Considering all data points (\# of bands $\times$ \# of clusters) the percentage of cases where models fit observations within errors using the starburst/alternative laws is: Padova-older (Po, 72/70), Padova (Pn, 75/75), Geneva-older (Go, 70/72), Geneva (Gn, 65/72), Geneva rotating (Gr, 57/57), Auckland (An, 67/70), and Auckland binary (Ab, $80 / 82$ ), where models assume single non-rotating stars, unless specified otherwise. This can be seen by integrating the results shown in Fig. 1, which for each cluster (different panels) and model (horizontal axis) gives the \# of bands where the residual in the magnitude is within the observational error. We use filled symbols for results obtained with the starburst attenuation law and unfilled symbols for results using an alternative law. It is not clear that the above success rates will hold under different assumptions of metallicity and attenuation. Considering the 6 clusters in NGC 1566 and all models, the percentage of cases where models fit individual bands within errors is: NUV $(64 / 69)$, U (76/76), B (29/31), V (86/90), and I (45/50). For NGC 5253, most bands are well fitted due to larger errors. With the starburst (alternative) laws, clusters span ranges of $E(B-V)=0.06 \pm 0.02$ to $0.49 \pm 0.15(0.05 \pm 0.02$ to $0.54 \pm 0.13) \mathrm{mag} ;$ mass $=1.6 \pm 0.3$ to $7.1 \pm 3.8(1.8 \pm 0.6$ to $8.9 \pm 7.3) \times 10^{4} M_{\odot}$; and age $=1.6 \pm 0.4$ to $40 \pm 27.7(2.6 \pm 0.6$ to $32 \pm 27.5)$ Myr (median \& standard deviation of all models). The typical scatter in properties derived with different models and starburst (alternative) laws is $\Delta E(B-V)=$ $\pm 0.09( \pm 0.09) \mathrm{mag}$, a factor of $3.6(3.9)$ in mass, \& a factor of 3.1 (2.5) in age.

\section{References}

Adamo, A. et al. 2015, to be subm.

Calzetti et al. 2000, ApJ, 533, 682

Calzetti, D., et al. 2015a, AJ, 149, 51

Charlot, S. \& Bruzual, G. 2015, to be subm. to MNRAS

Eldridge, J. J. et al. 2008, MNRAS, 384, 1109

Eldridge, J. J. 2015, to be subm.

Ferland, G. J. et al. 2013 RMxAA, 49, 137

Georgy, C., et al. 2013, A\& $A$, 558, A103

Gordon, K. D. et al. 2003, ApJ, 594, 279

Leitherer et al. 2014, ApJS, 212, 14

Mathis, J. S. 1990, ARAA, 28, 37

Tang, J. \& et al. , 2014, MNRAS, 445, 4287

Wofford, A. et al. 2015, to be subm. to MNRAS

Zackrisson, E. et al. 2011, ApJ, 740, 13 